\title{
INJECTING A KAPCHINSKIJ-VLADIMIRSKIJ DISTRIBUTION INTO A PROTON SYNCHROTRON*
}

\author{
E. Crosbie and K. Symon ${ }^{1}$ \\ Argonne National Laboratory, 9700 South Cass Avenue, Argonne, IL 60439 USA
}

\section{INTRODUCTION}

Recently it has been suggested that the KapchinskijVladimirskij (KV) distribution [1] may be of practical interest for high intensity machines in that it may provide the maximum space charge limit for such a machine. One can make a plausible argument that the maximum beam intensity is obtained for a distribution for which all particles have the same tune, at least when the resonance is approached. Therefore, the following steps should be taken: first, reduce the chromaticity of the accelerator ring as much as possible, and second, make the betatron frequencies independent of amplitude, i.e., make the focusing forces linear.

One way to make the focusing forces linear is to start with external focusing forces which are linear, and then make the space charge forces also linear by using a KV distribution [1]. Sections II and III describe two injection scenarios which produce a KV distribution (if we neglect beam-beam interactions during the injection process.) Simulations of these injection scenarios verify that the resulting distribution produces a uniform circular beam in $x y$-space.

A simulation code was written which also includes the space charge interactions between the 500 injected turns in the proposed scenarios; the results are given in section IV. The space charge forces have a substantial effect on the resulting distribution.

\section{PAINTING SCENARIO}

The KV distribution is essentially a microcanonical distribution with the beam distributed uniformly over a three-dimensional energy shell corresponding to a fixed total energy in the four-dimensional phase space of the $x$ and $y$ betatron oscillations. We need to construct a scenario which allows us to paint the energy shell uniformly.

Let us start from the simple Hamiltonian

$$
H\left(x, p_{x}, y, p_{y}\right)=\frac{p_{x}^{2}}{2}+\frac{v_{x}{ }^{2} x^{2}}{2}+\frac{p_{y}{ }^{2}}{2}+\frac{v_{y}{ }^{2} y^{2}}{2}
$$

Assuming that the focusing forces are linear, the space charge forces for the KV distribution will also be linear and

* Work supported by the U.S. Department of Energy, Office of Basic Energy Sciences, under Contract No. W-31-109-ENG-38.

'permanent address: Dę̧t. of Physics, University of Wisconsin-Madison, Madison. WI 53706 USA.

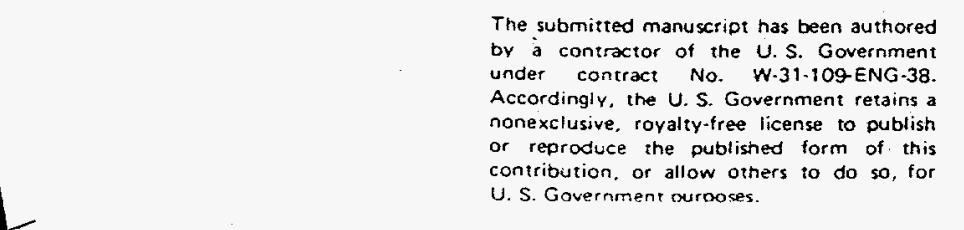

are included in the constants $v_{x}, v_{y}$. The independent variable is the angle $\theta=s / R$ around the ring. To simplify the algebra, $v_{x}, v_{y}$ are assumed to be constant. (For a more complete analysis, including a derivation of the $\mathrm{KV}$ distribution for the alternating gradient case with an elliptical beam, see Ref. [2].)

Introduce angle-action variables:

$$
\begin{array}{ll}
x=\left(2 J_{x} / v_{x}\right)^{1 / 2} \sin \gamma_{x}, & p_{x}=\left(2 v_{x} J_{x}\right)^{1 / 2} \cos \gamma_{x}, \\
y=\left(2 J_{y} / v_{y}\right)^{1 / 2} \sin \gamma_{y}, & p_{y}=\left(2 v_{y} J_{y}\right)^{1 / 2} \cos \gamma_{y},
\end{array}
$$

where the actions $J_{x}, J_{y}$ are constants of the motion.

The necessary KV distribution can be written in the form

$$
D\left(J_{x}, J_{y}, \gamma_{x}, \gamma_{y}\right)=A \delta\left(2 J_{x} \cos ^{2} \zeta+2 J_{y} \sin ^{2} \zeta-J_{0}\right)
$$

where $J_{0}$ is constant and $\zeta$ is an angle which may be chosen (almost) arbitrarily to change the aspect ratio of the elliptical beam boundary in xy space. If $\zeta=\pi / 4$ then this is a microcanonical distribution. If the distribution in Eq. (3) is written as a function of $x, y, p_{x}, p_{y}$ and integrated over $p_{x}$ and $p_{y}$, the result is a uniform distribution over the spatial coordinates $x, y$ within the ellipse

$$
v_{x} x^{2} \cos ^{2} \zeta+v_{y} y^{2} \sin ^{2} \zeta=J_{0} .
$$

Note that we can make the beam cross section circular by choosing

$$
\tan \zeta=\left(v_{x} / v_{y}\right)^{i / 2} .
$$

If the beam is injected at a fixed point in the phase space, the betatron oscillations will spread the beam over the $\gamma_{x} \gamma_{y}$ phase plane. In order to spread it over the threedimensional energy surface, the action variables need to vary in an appropriate way. To this end, the following variables are introduced:

$$
\begin{aligned}
& J_{0}=2 \cos ^{2} \zeta J_{x}+2 \sin ^{2} \zeta J_{y}, \\
& J_{m}=2 \cos ^{2} \zeta J_{x}-2 \sin ^{2} \zeta J_{y} .
\end{aligned}
$$

The Jacobian of this transformation is constant, so if area is conserved in the $J_{x} J_{y}$ phase plane then it is also conserved in the $J_{i} J_{\mathrm{m}}$ phase plane.

The total action $J_{0}$ is to be held constant and $J_{\mathrm{m}}$ is to be varied slowly. If the variation of $J_{\mathrm{m}}$ is slow compared with the betatron frequencies, then near each value of $J_{m}$ the betatron motion will distribute the injected beam uniformly over the $\gamma_{x} \gamma_{y}$ phase plane, provided there is no rational

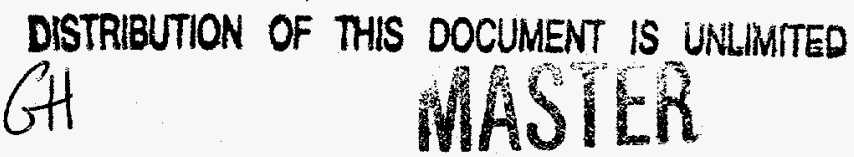




\section{DISCLAMMER}

This report was prepared as an account of work sponsored by an agency of the United States Government. Neither the United States Government nor any agency thereof, nor any of their employees, makes any warranty, express or implied, or assumes any legal liability or responsibility for the accuracy, completeness, or usefulness of any information, apparatus, product, or process disclosed; or represents that its use would not infringe privately owned rights. Reference herein to any specific commercial product, process, or service by trade name, trademark, manufacturer, or otherwise does not necessarily constitute or imply its endorsement, recommendation, or favoring by the United States Government or any agency thereof. The views and opinions of authors expressed herein do not necessarily state or reflect those of the United States Government or any agency thereof. 


\section{DISCLAIMER}

Portions of this document may be illegible in electronic image products. Images are produced from the best available original document. 
relation with small denominator between $\gamma_{x}$ and $\gamma_{y}$. In order to paint the $J_{0}$ shell uniformly, $d J_{\mathrm{m}} / d t$ is required to be constant:

$$
J_{m}=J_{0}\left(1-\frac{2 t}{T}\right),
$$

where $T$ is the total injection time. Note that we want to paint both positive and negative values of $J_{\mathrm{m}}$. Equation (7) is adjusted for the case in which $J_{\mathrm{m}}=J_{0}$ initially, i.e., the $x$ amplitude is maximum and the $y$ amplitude is zero. The injected $x, y$ actions are given by

$$
\begin{aligned}
& J_{x}=\frac{1}{2 \cos ^{2} \zeta} J_{0}\left(1-\frac{t}{T}\right), \\
& J_{y}=\frac{1}{2 \sin ^{2} \zeta} J_{0} \frac{t}{T} .
\end{aligned}
$$

This scheme is easily accomplished using stripping injection into a proton accelerator. The desired distribution is achieved using a local orbit bump to control the horizontal amplitude and a steering magnet in the injection beamline to control the vertical amplitude.

A program was written to simulate this scenario as applied to the IPNS Upgrade [3]. The injection time $T$ corresponds to 500 injected turns. The maximum injected amplitude is $50 \mathrm{~mm}$. The tunes are $v_{x}=6.81, v_{y}=5.73$. Figure 1 shows the resulting distribution in $x y$ space at the end of injection. Each of the small circles represents one injected turn. The spatial density is fairly uniform and has a circular cross section. Figure 2 shows the result for a scenario in which the painting is accomplished by using both horizontal and vertical orbit bumps at the stripper location. Since both orbits must move away from the stripper during injection, the resulting distribution cannot lie on the energy shell and is neither circular nor uniform.

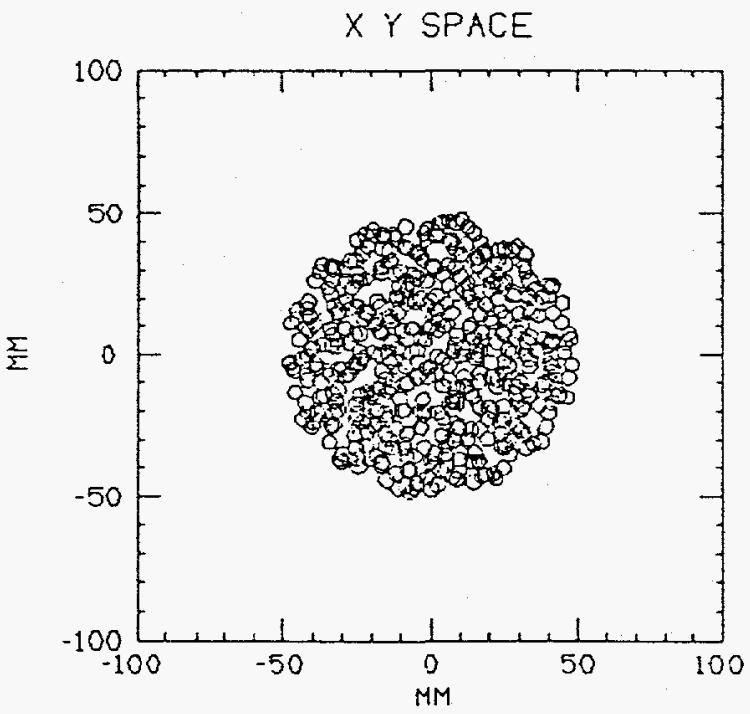

Fig. 1: Spatial distribution resulting from the painting scenario.

\section{COUPLING SCENARIO}

Cho [4] has suggested an elegant, practical way to produce a KV distribution. He proposes making the $x$ and $y$ betatron tunes equal, providing a small coupling between them, and then injecting with zero $y$ amplitude and at a large fixed $x$ amplitude. The coupling causes the $y$ oscillation energy to increase at the expense of the $x$ energy. This has two effects. First, it causes the previously injected beam to move away from the inflector and remain away for one beat period, thus permitting multiturn injection. Second, it results in a distribution in which all particles have the same total oscillation energy.

In order to fill the entire energy shell a careful analysis is required similar to that in the previous section. A suitable scenario has been derived; the details can be found in Ref. [2]. Simulations again show that this scenario can achieve a KV distribution with a uniform spatial density within a circular beam.

\section{SIMULATIONS INCLUDING SPACE CHARGE FORCES}

A simulation code which includes the effects of the forces exerted by the 500 injected turns on one another has also been written. In this simulation each injected turn is represented as a tube of charge. Each tube exerts forces on all other tubes that are present in the machine during the injection process. The forces are inversely proportional to the distances between the tubes for separations larger than the tube sizes. For separations smaller than the tube sizes, the forces vary linearly with the separation. If one first injects the bearn and turns on the space charge forces after all 500 turns have been injected, the resulting distribution of tune shifts is in good agreement with the expected results and shows clearly the advantage of injecting with a uniform spatial distribution.

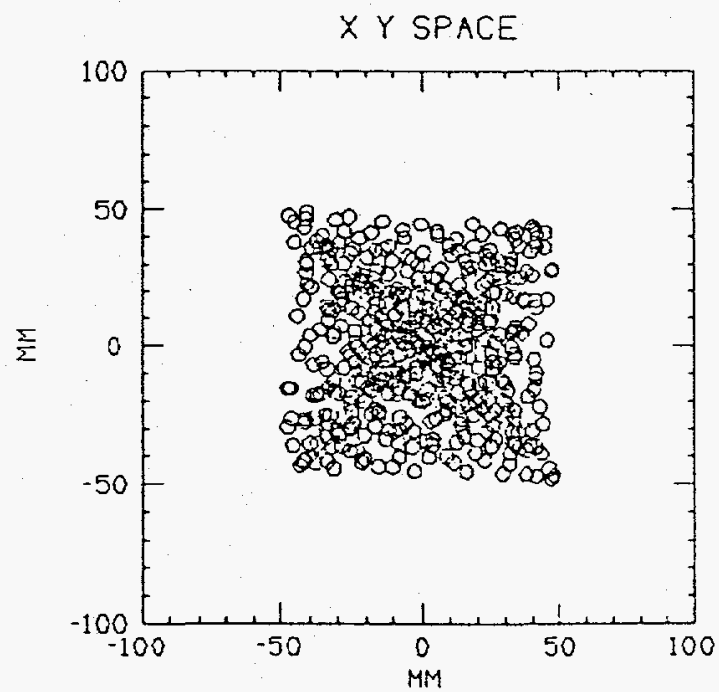

Fig. 2: Spatial distribution resulting from a non-KV injection. 
If, more realistically, we include the space charge forces during the injection process, we do not reach a true KV distribution. This can be seen in Fig. 3, which shows the final distribution after injection with the painting scenario. The total injected current is about half of the calculated space charge limit for a uniform bean cross section. The space charge forces substantially affect the resulting distribution. (Compare Fig. 3 with Fig. 1.) However, the distribution is still fairly uniform over the nearly circular cross section. Figure 4 presents a similar calculation with a current two times the calculated space charge limit. The space charge forces have tuned the beam to the half-integral resonance, resulting in increased beam size and reducing the beam density enough to prevent the tunes from remaining in the stop band.

With a beam intensity exceeding the space charge limit we are able to see clearly the effect of the approach to the resonance, particularly in the tunes of the beam particles which tend to remain near the edge of the stop band. When the magnet error which drives the bump is small, and we inject a large beam, say four times the space charge limit, the resonant growth rate may not be large enough to prevent the beam from crossing the resonance. However the resulting beam cross section is not much different. If we turn off the gradient error which drives the resonance, the tunes cross the resonance and end up well below it, but the beam cross section is still not much different. We conclude that the beam-beam interactions dominate the final beam distribution.

If we inject using a non-KV scenario, the effect of the approach to resonance is to modify the distribution so as to produce a beam more uniform in spatial cross section than would otherwise result. The non-KV scenario differs in that the tune may approach resonance much sooner during the injection process, but the final result is not much different from the KV scenario. We conclude that after almost any injection scenario a beam exceeding the space charge limit will tend to adjust its distribution to produce a fairly uniform cross sectional distribution. There seems to be little advantage in using a scenario intended to produce a $\mathrm{KV}$ distribution.

\section{REFERENCES}

[1] I. M. Kapchinskij and V. V. Vladimirskij, "Limitations of Proton Beam Current in a Strong Focusing Linear Accelerator Associated with the Beam Space Charge," Proc. CERN Symposium on High Energy Accelerators, 1959.

[2] K. R. Symon, "Kapshinskij-Vladimirskij Distribution," Argonne National Laboratory Neutron Source Accelerator Note NSA-95-5 (available on request).

[3] "IPNS Upgrade - A Feasibility Study," ANL-95/13, April 1995.

[4] Y. Cho, private communication.

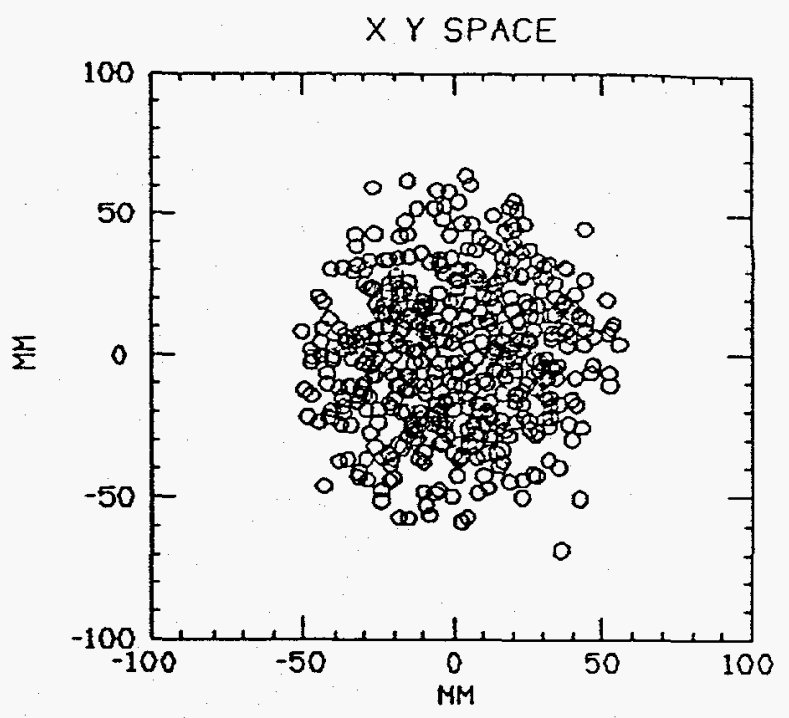

Fig. 3: Spatial distribution including the effect of space charge forces (painting scenario).

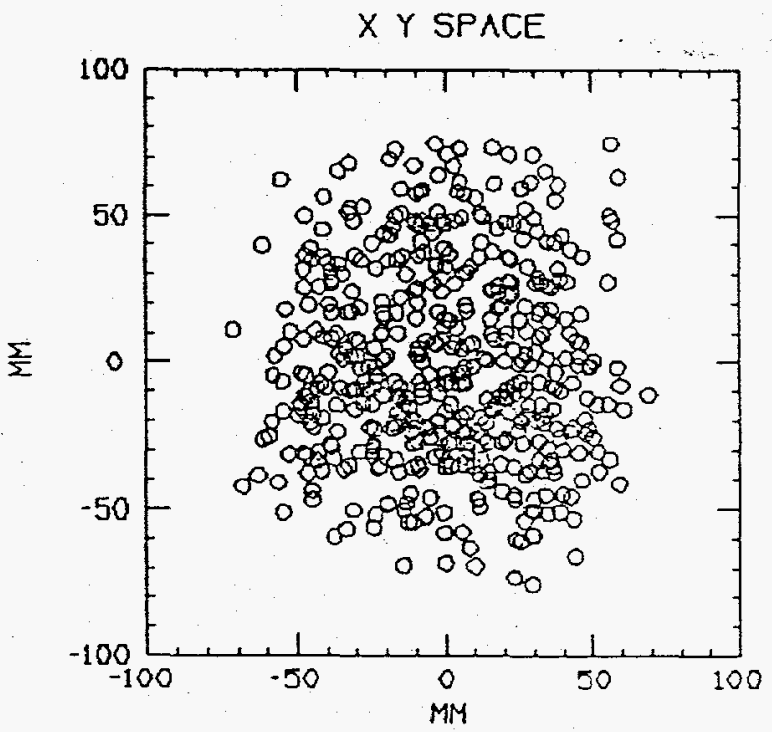

Fig. 4: Spatial distribution with space charge forces for a beam above the space charge limit. 